\title{
De la toxicomanie aux addictions ou les deux âges de la drogue contemporaine
}

«Pour se plaire à une drogue, il faut aimer être sujet. Moi, je me sentais trop de corvée 》 Henri Michaux, Misérable miracle, 1955.
Sociologue, CESAMES, Centre

sont promus comme des moyens chimiques pour explorer l'espace du dedans et inventer d'autres rapports humains, combinant ainsi une mystique (« Je veux voir Dieu en face », fut l'un des slogans du LSD) et une politique - La politique de l'extase, pour reprendre le titre du livre de Timothy Leary, professeur de psychologie à Harvard et « pape »du LSD. Expansion de la conscience est le motclé. C'est le temps du feeling. L'usage de drogues concentre ainsi les utopies émancipatrices de l'époque qu'elles radicalisent; elles symbolisent les tendances nouvelles, en Occident, à lever les interdits empêchant chacun à disposer de lui-même comme il l'entend. Elles incarnent la volonté de faire table rase des valeurs de la famille, de l'autorité, du travail aliénant et de la réussite sociale. C'est donc la question des limites à la propriété de son propre corps qui, au-delà des risques sanitaires, est posée par la drogue. C'est effectivement en termes de transgression de la loi, et non de risques, pour la santé, que la lutte contre la drogue s'organise en France.

\section{De l'interdit ...}

Trois éléments se sont cumulés pour forger la politique française de lutte contre les drogues qui émerge en 1970 : la culture politique républicaine qui imprègne l'action publique, les conceptions du soin par le corps professionnel qui a obtenu le monopole de gestion de ces questions, l'absence de politique de santé publique [5]. La définition politique de la drogue est liée à la façon dont la culture politique républicaine conçoit la citoyenneté. Ce style politique est lié à une vision générale des rapports État-société qui conduit à employer la loi moins pour régler des pratiques diversifiées que pour fixer des interdits et des normes : c'est le cas de la loi du 31 décembre 1970 qui étend à l'usage privé l'interdit 
pénal limité depuis la loi du 2 juillet 1916 à l'usage public. Cette politique se caractérise par un triangle d'or : l'abstinence comme idéal normatif, la désintoxication comme stratégie, l'éradication des drogues de la société comme horizon politique. En France, la drogue concerne d'abord le statut de citoyen. Le législateur a considéré que la société doit imposer des limites à I'utilisation que chacun peut faire de son propre corps en contrepartie du droit à la santé et aux soins instaurés par la Sécurité sociale. Or, il n'est guère habituel de trouver dans la loi pénale le moyen principal de promouvoir une politique de santé publique. Les parlementaires sont d'ailleurs conscients du caractère exceptionnel de cette loi du point de vue des libertés individuelles [6]. Définissant le drogué à la fois comme prisonnier de sa liberté (malgré les avis pourtant fort nuancés des psychiatres et des policiers auditionnés comme experts par le Parlement) et danger pour l'ordre public, la drogue est ainsi tendue entre trouble mental et délinquance. L'injonction thérapeutique, qui permet au drogué d'échapper aux foudres du pénal, grâce à la prise en charge thérapeutique, opère un compromis entre les deux aspects du problème.

Par conséquent, la loi de 1970 est une loi typiquement républicaine : le drogué est celui auquel on doit rappeler qu'il vit en société avant de l'empêcher de s'autodétruire. Le style politique français traite une question collective comme un ensemble de cas individuels, instaurant une confusion entre registre clinique et registre politique. La loi flotte entre légalité républicaine - trouble à l'ordre public - et clinique - trouble du rapport à soi. Cette conception conduit à lutter contre la drogue à partir de la justice et de la psychopathologie, comme s'il n'existait aucune autre médiation entre le sujet et la loi. Or, les régulations institutionnelles ont une efficacité limitée. De plus, il existe d'autres formes de régulation (autocontrôles, pressions sociales) [7].

Dans la foulée de la loi de 1970 et de l'injonction thérapeutique est créé le secteur spécialisé qui obtient le monopole de soin des drogués. Les travailleurs sociaux et les psychiatres qui en ont la charge se réfèrent à un double modèle : l'anti-psychiatrie - qui permet une relation d'empathie, allant parfois jusqu'au fusionnel, avec leur clientèle - et la psychanalyse, qui implique que le patient se présente avec une demande libre d'où leur hostilité à l'injonction thérapeutique. Ce double modèle constitue le soubassement de prises en charge thérapeutiques visant moins à pratiquer des psychanalyses de toxicomanes, restées très marginales, qu'à mettre en place des accompagnements non coercitifs ancrés dans une problématique de l'inconscient. Les intervenants en toxicomanie sont divisés face à l'inter- dit pénal et généralement défavorables à la réduction de la prise en charge à la désintoxication (la drogue n'est pas une maladie, mais un symptôme) comme à la médicalisation de la drogue (considérée comme une forme de contrôle social). Ce double modèle représente pour ces acteurs une ressource stratégique permettant d'éviter deux dangers : l'enfermement des toxicomanes dans les prisons ou leur internement en hôpital psychiatrique (jusqu'aux années 1960, les toxicomanes, souvent diagnostiqués comme psychopathes incurables ou pervers, se retrouvaient dans les hôpitaux psychiatriques). Bref, la drogue et la toxicomanie sont moins affaires de médecine et de santé publique que de trouble de la personnalité - ce qui, bien entendu, n'est pas faux, mais ne peut en aucune manière définir la totalité du problème.

Le troisième élément est l'absence de politique de santé publique. Le risque de la contamination par le sida aurait dû avoir pour conséquence de redéfinir une partie du traitement des usages de drogues - ceux qui passent par l'injection intraveineuse - dans une perspective plus médicale et déboucher sur une diversification de l'offre de soins. Ce changement de paradigme s'avérait d'autant plus nécessaire que les multiples pathologies somatiques des consommateurs abusifs de cocaïne et d'héroïne les plus désocialisés sont peu traitées par le système sanitaire français. Mais les autorités sanitaires et la médecine française n'ont guère été préparées à accueillir l'arrivée inopinée du VIH. Car la drogue souffre également d'un problème qui ne lui est absolument pas spécifique : s'il existe bien entendu des actions de santé publique, on ne peut pourtant parler de politique de santé publique. Cette absence a conduit, dans le cas de la drogue comme dans celui de la transfusion sanguine ou de la politique de dépistage, à sous-évaluer systématiquement le risque sida (comme celui des hépatites B et C). L'histoire du système de santé montre que la France n'a jamais élaboré une véritable politique de santé publique et qu'elle est dominée par le modèle curatif de la clinique individuelle ([8] et, pour une analyse historique sur un siècle [9]).

Le triangle d'or abstinence-désintoxication-éradication ne conduit pas la République à se demander comment protéger l'individu de lui-même pour éviter qu'il ne s'autodétruise. Elle lui rappelle qu'il doit se conformer à la norme civique ou, à défaut, être soigné ou puni.

\section{... au risque}

Le système français évolue néanmoins sur le plan sanitaire à la fin des années 1980 et, surtout, des dernières 
années de la décennie 1990 avec l'amorce de modifications pratiques notables: mise en vente libre des seringues en 1987, développement de programmes de substitution depuis 1993, autorisation de mise sur le marché de la méthadone et de la buprénorphine en 1995, décision de créer dans chaque département un centre de distribution de produits de substitution. L'émergence de nouveaux acteurs de soin (associations de lutte contre le sida, Médecins du monde et Médecins sans frontières), l'influence croissante de nouveaux milieux de recherche en ce domaine (neurobiologistes et pharmacologues qui redéfinissent la drogue comme une maladie chronique du cerveau $[10,11]$ ) et le recours croissant d'héroïnomanes à la médecine générale contribuent à redéfinir la politique de la lutte contre les drogues en termes de risques à réduire. La circulaire du 31 mars 1995 officialise le tournant en autorisant les médecins généralistes à délivrer des produits de substitution [12]. Le cadre cognitif et les croyances traditionnelles en matière de toxicomanie changent [13]. À la référence au couple toxicomanieinterdit, associée à une perspective de guérison ayant pour horizon l'abstinence tend à se substituer la référence à une polarité addiction-risque, associée à un accompagnement de longue durée : la dépendance aux drogues est désormais considérée comme une maladie chronique à laquelle répondent des programmes de maintenance visant à assurer une bonne qualité de vie aux patients - sur le modèle traditionnel des maladies chroniques en médecine et des psychoses en psychiatrie. À un type de personne pathologique que l'on espérait guérir succède un type « personne dysfonctionnelle » que l'on entend soutenir. C'est la fin du triangle d'or. II s'agit désormais de hiérarchiser les risques en tenant compte de la dangerosité réelle des drogues. Ce déplacement sera facilité par la publication d'un rapport établi par un pharmacologue de renom [14].

La nouvelle politique est officialisée dans le Plan triennal (1999-2001) de la Mission interministérielle de lutte contre la drogue et la toxicomanie : « L'évolution des modes de consommation, notamment chez les jeunes (polyconsommation, fragmentation des lieux et des modes d'usage, consommation de drogues de synthèse associées à l'alcool, au tabac, aux médicaments ou au cannabis, usage détourné de médicaments, prise concomitante de produits dopants, extrême fréquence du recours à plusieurs produits) conduit à rechercher une approche qui tienne compte de l'ensemble des conduites addictives, quel que soit le statut juridique du produit concerné » [15]. Un cadre administratif et financier global est instauré, quel que soit le statut juridique des substances. La distinction entre usage, abus et dépendance, mise en avant par les cliniciens depuis longtemps, est au cœur de la nouvelle politique, centrée sur une approche globale des conduites à risque et des comportements de dépendance (on trouvera une synthèse sur l'évolution des dispositifs et des conceptions de la prise en charge dans [16]).

Un diplôme d'études spécialisées en « addictologie » est créé et les addictions deviennent un domaine à part entière. Si la loi de 1970 représente un véritable fétiche auquel aucun gouvernement ne veut toucher, sous peine de paraître approuver l'usage de drogues cela malgré l'avis convergent de la plupart des experts (cliniciens et biologistes) et malgré les modifications législatives votées dans plusieurs pays européens (Pays-Bas, bien sûr, mais aussi Suisse, Italie, Allemagne, Espagne [17]) -, il est indéniable que la politique française a changé [18].

\section{Se droguer, se soigner, se doper ou la reconfiguration de la drogue}

Cette nouvelle politique se développe dans un contexte où le statut médical et social des médicaments psychotropes (anxiolytiques, hypnotiques, antidépresseurs) connaît de profonds changements. Ces médicaments entrent dans le débat public au cours des années 1970 via le thème du contrôle social par l'état, le corps médical et l'industrie pharmaceutique, alliés pour faire marcher ensemble le profit et la tranquillité sociale. Le débat se déplace au cours de la deuxième moitié des années 1980. La publication à l'été 1988 d'un Guide des 300 médicaments pour se surpasser physiquement et intellectuellement provoqua des réactions scandalisées, parce qu'il était un plaidoyer pour le «droit au dopage »dans une société de compétition exacerbée. Au début de l'année suivante, un rapport signé par cinq professeurs de médecine critique la surconsommation et le mésusage des anxiolytiques et des hypnotiques. Les controverses reprennent au cours des années 1990, avec le lancement d'une nouvelle classe d'antidépresseurs (les inhibiteurs spécifiques de la recapture de la sérotonine). Leurs effets secondaires réduits et leur toxicité faible ainsi que leur facilité d'emploi en font un médicament d'élection pour la médecine générale (auparavant rétive à prescrire ces molécules) et permettent d'étendre le traitement médicamenteux aux dépressions dont le degré de sévérité est faible, y compris les cas sub-syndromiques. Prescrits, selon certains, à tort et à travers, ces médicaments serviraient plus au confort des consommateurs, voire à l'amélioration de 
leurs performances sociales qu'à leur santé. Les controverses se déroulent entre les trois pôles du soin, du confort et du dopage. Elles se caractérisent par une remarquable confusion parce que le statut du trouble mental a profondément changé en trente ans sans qu'un effort conceptuel ait été entrepris pour éclairer ce changement : associé à la «folie » au début des années 1970, le trouble mental s'est désormais diversifié en une multitude de troubles de la subjectivité individuelle regroupés dans la catégorie «souffrance psychique $\gg$.

Ces controverses jouèrent un rôle catalyseur pour remettre en cause deux frontières jusque-là acceptées : l'extension de la notion de drogue à ces médicaments (on peut se «droguer » en les ingérant) et leur sortie du champ de la pathologie vers celui de la consommation, via le thème du confort, ou celui de la socialisation, via le thème du dopage. Les médicaments psychotropes sont, en France, les substances révélatrices d'un déplacement des représentations des usages de la drogue : ils tendent à se transformer en moyens d'augmenter ses performances et son confort psychique. Ces «drogues » se présentent comme un adjuvant chimique permettant de démultiplier ses propres possibilités dans des formes de socialité exigeant moins de l'obéissance et de la discipline que de l'initiative et de l'action, d'une part, et moins de s'adapter vaille que vaille à un destin que de choisir sa vie dans une perspective d'épanouissement personnel, d'autre part. Le brouillage des frontières entre drogues illicites et psychotropes prescrits est aujourd'hui une caractéristique structurelle, et il est clair que nous ne reviendrons pas en arrière. L'extension de l'usage du mot « dopage » hors du sport est une manière d'enregistrer confusément ce brouillage. On n'est ni dans la maladie (le couple normal-pathologique) ni dans la transgression (le couple permis-défendu), mais dans l'amélioration de soi, la démultiplication de ses propres possibilités dont il faut tester les limites et mesurer les risques.

L'usage massif de substances diverses se présente comme un corrélat de la culture de l'initiative individuelle généralisée et de la revendication identitaire totale (« c'est mon choix ») au sein de laquelle nous sommes désormais voués à vivre. Dans une forme de vie où la règle sociale consiste à décider et à agir par soi-même, la désinhibition est une condition de la socialisation que les substances psychotropes facilitent. Les addictions relèvent certes pour partie de la pathologie, mais sociologiquement elle sont une composante de nos modes de vie. Elles sont baignées dans notre horizon normatif. $\diamond$

From illegal drug addiction to addiction or the two ages of contemporary drug

\section{RÉFÉRENCES}

1. De Quincey T. Les confessions d'un mangeur d'opium anglais. Paris : Gallimard, 1970.

2. Baudelaire C. Les paradis artificiels. Collection Folio. Paris : Gallimard, 1970.

3. Retaillaud-Bajac $\varepsilon$. Usages et usagers de drogues dans la France de l'entre-deux guerres, 2 vol. Thèse pour le Doctorat en Histoire, Université d'Orléans. 2000.

4. Musto DF. The American disease : origins of narcotic control, édition remaniée et augmentée. NewYorkOxford : Oxford University Press, 1987.

5. Ehrenberg A. L'individu incertain. Paris : CalmannLévy, 1995 (réedition Hachette-Pluriel, 1996).

6. Bernat de Celis J. Fallait-il créer un délit d'usage illicite des stupéfiants? Paris : CESDIP, n54, 1992.

7. Ogien A. Sociologie de la déviance et usages de drogues. Une contribution de la sociologie américaine. Documents du Groupement de recherche Psychotrope, Politique, Société, $n^{\circ} 5$, avril-juin 2000.

8. Setbon M. Pouvoirs contre sida. Paris : Seuil, 1993.

9. Murard L, Zylberman P. L'hygiène dans la République. La Santé publique en France ou l'utopie contrariée, 1870-1918. Paris : Fayard, 1996.

10. Koob GF, Le Moal M. Drug abuse : hedonic homeostatic dysregulation. Science 1997 ; 278 : 52-8.

11. Leshner Al. Addiction is a brain disease, and it matters. Science $1997 ; 278$ : 45-6.

12. Lert F. Méthadone, Subutex ${ }^{\circledR}$. Substitution ou traitement de la dépendance à l'héroïne? Questions en santé publique. In : Ehrenberg A. (sous la direction de). Drogues et médicaments : le trouble des frontières. Paris : Éditions Esprit, 1998.
13. Bergeron H. L'État et la toxicomanie : histoire d'une singularité française. Paris: PUF, 1999.

14. Roques B. La dangerosité des drogues. Paris : Odile Jacob-La Documentation Française, 1999.

15. MILDT. Plan triennal de lutte contre la drogue et de prévention des dépendances, 1999.

16. Morel A, Favre JD, Rigaud A. Rapprocher l'alcoologie et l'intervention en toxicomanies? Alcoologie et Addictologie 2001 ; 23 : 393-403.

17. Cesoni ML. L'incrimination de l'usage de stupéfiants dans sept législations européennes. Documents du Groupement de recherche Psychotropes, Politique, Société, $n^{\circ} 4$, janvier-mars 2000.

18. Faugeron C, M. Kokoreff (sous la direction de). Société avec drogues : enjeux et limites. Ramonville Sainte-Agne : Érès, 2002.

\section{TIRÉS À PART}

A. Ehrenberg 